\title{
Análisis de la arquitectura de webs mediante tests de estrés de navegación, de usabilidad y eye tracking
}

\author{
Por Juan-Miguel López-Gil, Carolina Navarro-Molina, Roberto García y Rafael Aleixandre-Benavent
}

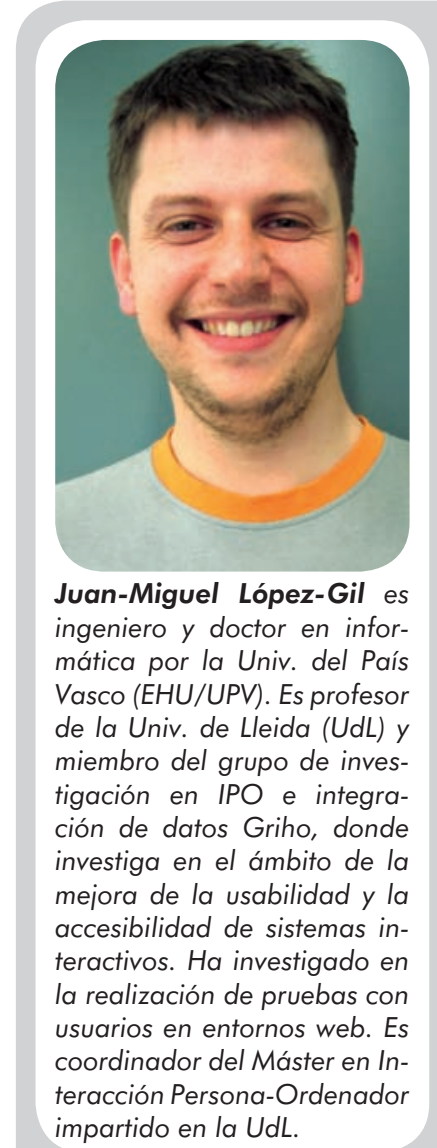

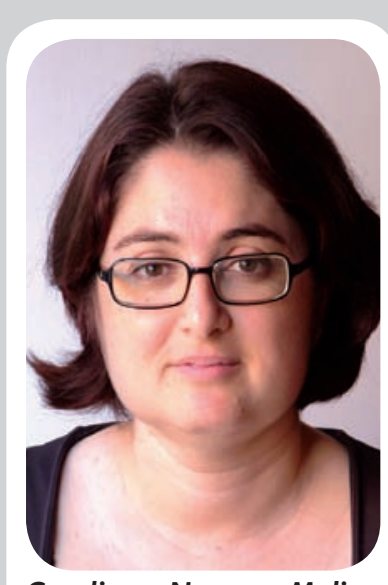

Carolina Navarro-Molina es licenciada en documentación por la Universidad Politécnica de Valencia (UPV). Sus principales líneas de investigación se enfocan a los distintos aspectos de la interacción persona-ordenador relacionados con la comunicación científica. Se encuentra realizando su tesis doctoral sobre revistas electrónicas médicas y criterios de calidad formal.

http://www. directorioexit. info/consulta.php? directorio $=$ exit\&texto $=2000$

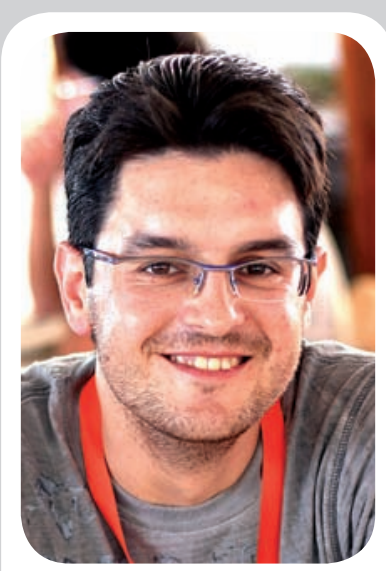

Roberto García es ingeniero en informática por la Univ. Politècnica de Catalunya (UPC), Máster en Comercio Electrónico por La Salle Barcelona y doctor por la Univ. Pompeu Fabra (UPF). Es profesor de la Univ. de Lleida (UdL) y miembro del grupo de investigación en IPO (Interacció Persona Ordenador) e integración de datos Griho (Grup de Recerca en Interacció Persona Ordenador i Integració de Dades), donde investiga cómo hacer llegar la web semántica a los usuarios. http://rhizomik.net/ roberto

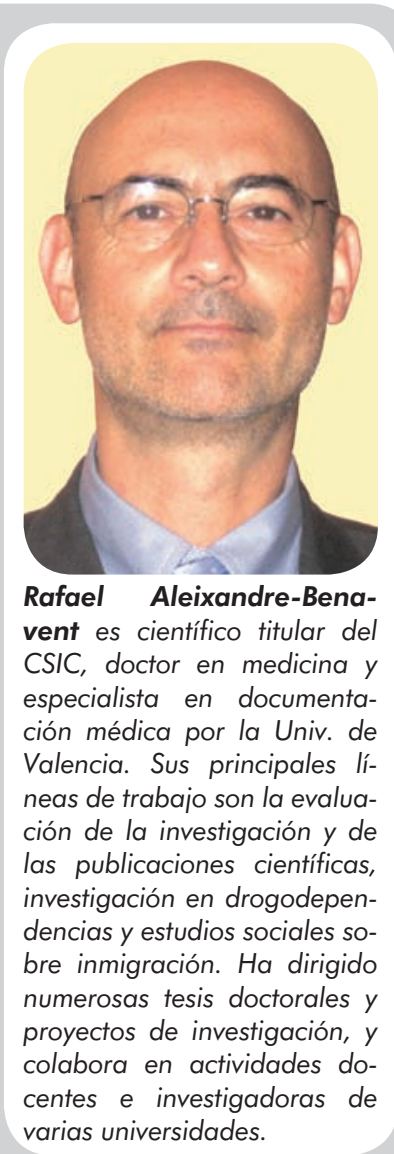

Resumen: Evaluar una arquitectura de la información en un sitio web ya desplegado no resulta una tarea sencilla. La mayoría de las técnicas se centran en examinar la usabilidad del sistema que, aunque afecta a la arquitectura de la información, no es el único factor que influye en ella. La principal técnica que se utiliza es el test de estrés de navegación. Se muestra un aporte metodológico para hacer dicha técnica más informativa, llevándola más allá de la simple anotación en papel por parte del usuario de respuestas a las preguntas de navegación planteadas. Se propone la combinación de ésta con otras técnicas de evaluación de la usabilidad: la técnica de pensar en voz alta o thinking aloud y un cuestionario de usabilidad. Se ha utilizado un sistema de seguimiento de la mirada o eye tracking para complementar la información obtenida mediante las técnicas aplicadas. El enfoque metodológico planteado se ha puesto a prueba analizando una serie de sitios web de bibliotecas de universidades públicas españolas. Se muestra en los resultados la validez del enfoque empleado, así como el valor que dicho enfoque y el uso del eye tracking aportan al análisis de la arquitectura de la información respecto al test de estrés de navegación tradicional.

Palabras clave: Arquitectura de la información, Pruebas con usuarios, Thinking aloud, Test de estrés de navegación, Test de usabilidad, Evaluación, Eye tracker, Eye tracking.

Title: Analysis of web-based information architecture using a combination of navigation stress test, usability testing and eye tracking

Abstract: Evaluating the Information Architecture in an already deployed website is not an easy task. Most evaluation techniques are focused on examining system usability, but this is not the only factor that influences IA. The main technique that deals with IA in already deployed web environments is the Navigation Stress Test (NST). A new methodology makes this technique more informative by taking NST beyond simple notation on paper. This work proposes the use of NST combined with other usability testing techniques such as Thinking Aloud and a usability questionnaire. Eye tracking also has been used to supplement the information obtained from applied techniques. This new methodology has been tested by analyzing a series of websites belonging to Spanish public university libraries. The results of this study show the validity of 
the approach used, as well as the value that this approach and the use of Eye tracking bring to the analysis of IA compared to traditional NST.

Keywords: Information architecture, User tests, Thinking aloud, Navigation stress test, Usability tests, Evaluation, Eyetracker, Eye tracking.

López-Gil, Juan-Miguel; Navarro-Molina, Carolina; García, Roberto; Aleixandre-Benavent, Rafael. "Análisis de la arquitectura de webs mediante tests de estrés de navegación, de usabilidad y eye tracking". El profesional de la información, 2010, julio-agosto, v. 19, n. 4, pp. 359-367.

DOI: $10.3145 /$ epi.2010.jul.04

\section{Introducción}

Uno de los factores más importantes en el diseño de un sitio web es la correcta construcción de su arquitectura de la información (IA). Esta disciplina se centra en el modo en el que se estructura la información en la web, lo que tiene una gran influencia en la forma en que los usuarios acceden e interaccionan con ella (Rosenfeld; Morville, 2006). Pero este no es el único factor a tener en cuenta en los sistemas interactivos, y a menudo la IA queda enmascarada detrás de los mecanismos utilizados para proporcionar interactividad al sistema, por lo que resulta complicado realizar la evaluación de manera independiente, determinar la raíz de los problemas de usabilidad detectados y ofrecer oportunidades de mejora.

Existen muchas técnicas para evaluar la IA pero también se ven influidas en gran medida por aspectos de interacción, como por ejemplo el método de thinking aloud (TA) o pensar en voz alta (Nielsen, 1993), el recorrido cognitivo (Blackmon et al., 2002) o los diversos cuestionarios empleados en la evaluación de la usabilidad (Nielsen, 1993). Estas técnicas están enfocadas a la evaluación de sistemas interactivos en general, es decir, no son específicas para la IA.

La evaluación de la IA de las webs de las bibliotecas ha sido incluida en numerosas ocasiones como un factor asociado con la navegabilidad de la página (López-Marín; Méndez; Sorli-Rojo, 2002), la presentación de la información (Patalano, 2002) (Melo-Alves; Quiroa-Herrera, 2007) o con su usabilidad (VílchezRomán, 2004) (Travieso-Rodríguez; Alonso-Arévalo; Vivancos-Sevilla, 2007). En los trabajos citados se ha empleado de forma preferente la evaluación heurística, pese a la existencia de métodos propios o más específicos para el análisis de la IA (Pérez-Montoro, 2010),

Existe una serie de técnicas empleadas habitualmente a la hora de diseñar la IA de sistemas de información, como la ordenación de tarjetas (card sorting), o las basadas en modelos mentales de los usuarios, pero no están concebidas para evaluar una web ya existente. A lo sumo se podrían comparar la existente y la nueva utilizando por ejemplo card sorting, aunque esta técni- ca sólo permitiría evaluar una pequeña parte de la IA, básicamente los esquemas de organización que sigan una estructura jerárquica. Otros aspectos fundamentales, como la forma de desplegar los elementos de la IA en la web o la presencia de sistemas de navegación contextuales, quedan fuera de su alcance, sin mencionar el elevado coste de dichos métodos comparado con la alternativa presentada a continuación ya que suponen un rediseño más que una evaluación.

En este sentido, la técnica más enfocada al estudio de la IA en sistemas de información ya desplegados y que proporciona mayor grado de independencia es el test de estrés de navegación (TEN) (Instone, 2010). Se basa en una serie de preguntas que determinan si una IA, tal y como se plasma en un sistema de información, permite a los usuarios hacerse una idea clara de dónde están y qué elementos de información tienen a su alcance desde ese punto.

Esta técnica presenta sin embargo una serie de limitaciones. En primer lugar, al tener que responder en papel a las cuestiones planteadas sobre la IA, y pensando específicamente en entornos web, se pierde información contextual que los usuarios emplean habitualmente para discernir aspectos relacionados con la IA. Por ejemplo, que el navegador marque el destino de los hiperenlaces al pasar el ratón por encima de ellos, o que el uso del navegador permita analizar cómo el scroll puede incidir en la percepción que los usuarios tienen sobre la IA de un sitio web. El TEN carece de mecanismos para discernir las causas de los errores que los usuarios puedan cometer a la hora de responder a las preguntas planteadas. Estos factores nos llevan a plantearnos la necesidad de emplear el TEN en combinación con otros métodos para obtener información más completa.

El eye tracking (ET) -seguimiento de los ojos- es una técnica novedosa que permite tener constancia de qué punto del sistema de información ha estado mirando el usuario en cada momento, la dificultad para encontrar aspectos concretos de la interfaz y el tiempo o el número de elementos analizados antes de determinar el adecuado. Esto permite que los evaluadores puedan saber si una de las causas de que los usuarios 
no perciban correctamente la IA es que no hayan fijado la mirada en algunos de los elementos relevantes de la misma. Lo más próximo son los trabajos utilizando ET sobre navegación e IA realizados por Nielsen y Pernice (2009).

El objetivo de este trabajo es evaluar la arquitectura de la información (IA) de un grupo de webs universitarias españolas empleando una combinación de técnicas: test de estrés de navegación (TEN) -específica del análisis de la IA-, thinking aloud (TA) y un cuestionario de usabilidad, así como eye tracking (ET) para la obtención de información suplementaria.

\section{"El empleo de la metodología planteada supone una nueva manera de evaluar} la IA"

\section{Material y métodos}

Se ha analizado la IA de 17 webs universitarias españolas. Para la selección de la muestra, se incluyó una universidad pública por comunidad autónoma, empleando como criterio de selección en el caso de haber más de una, el ranking de universidades elaborado por Webometrics. Las universidades analizadas aparecen en la tabla 1.

http://www.webometrics.info/rank_by_country_es.asp? country $=e s$

Fueron analizadas 4 páginas de las webs de cada universidad que se correspondían con el catálogo, préstamo interbibliotecario, carta de servicios y acceso remoto a la biblioteca. Dado que no todas las universidades seleccionadas disponían de esas funciones, el número de páginas de la muestra quedó finalmente compuesto por 54.

Para la selección de los usuarios se empleó un cuestionario demográfico en el que se recogieron edad y sexo, así como cuestiones relacionadas con el uso de internet y servicios de bibliotecas universitarias. Dicha información se empleó para la clasificación por perfiles de los usuarios que realizaron la prueba.

Han participado en el estudio un total de 6 personas (4 hombres y 2 mujeres) con una media de edad de 31 años. Todos ellos navegan por internet con una frecuencia diaria y en la mayoría de los casos disponen de conexión en su hogar. Si bien emplean servicios de bibliotecas, lo hacen de manera muy esporádica (no más de una vez al mes). Ninguno de ellos tenía experiencia en el uso de ninguno de los sistemas analizados.
Para evaluar la IA se empleó un cuestionario basado en el TEN. Los usuarios debían responder a cada una de las preguntas planteadas en él (Instone, 2010) para cada una de las webs analizadas:

- ¿Sobre qué es esta página web?

- ¿Qué sitio es éste?

- ¿Cuáles son las secciones más importantes del sitio?

- ¿En qué sección principal está la página?

- ¿Hay un nivel por encima de la página?

- ¿Cómo llegar a la página de inicio del sitio?

- ¿Cómo se llega a la página web principal de la sección?

- ¿Qué representa cada grupo de enlaces?

a) Enlaces que aportan más detalles, llevan a subpáginas.

b) Enlaces a páginas cercanas, dentro de la misma sección que esta página pero no subpáginas.

c) Enlaces a otras páginas del sitio pero no relacionadas, en otras secciones.

d) Enlaces a webs externas, fuera del sitio.

- ¿Cómo llegar desde la página de inicio del sitio a ésta?

Para mejorar la cantidad y calidad de la información obtenida mediante esta técnica, en este trabajo se ha combinado con otras típicas de la evaluación de la usabilidad y con la aplicación de un ET, lo que permite grabar toda la sesión para su posterior análisis y registrar el lugar en el que el usuario ha estado fijando su atención.

Los usuarios debían rellenar un cuestionario con el que se pretendía obtener una medida de la usabilidad percibida para cada sitio web analizado. Dicho cuestionario se basó en el system usability scale (SUS) (Brooke, 1996). Cada pregunta debía ser valorada en una escala de 0 a 4 , siendo 0 total desacuerdo y 4 total acuerdo. Mediante una fórmula matemática basada en los valores de las respuestas asignadas por los usuarios se obtiene un valor numérico de 0 a 100 que indica el nivel de usabilidad del sistema. En este trabajo se empleó el SUS para relacionar la IA percibida por los usuarios sobre los sitios web con la usabilidad percibida sin necesidad de que estos interactuaran con el sistema, dado que dicha interacción hubiera condicionado los resultados del análisis de las preguntas de navegación relacionadas con la IA de las webs analizadas. Dichas técnicas se combinaron para que los usuarios evaluaran la usabilidad de los sistemas cuya IA se estaba analizando. 


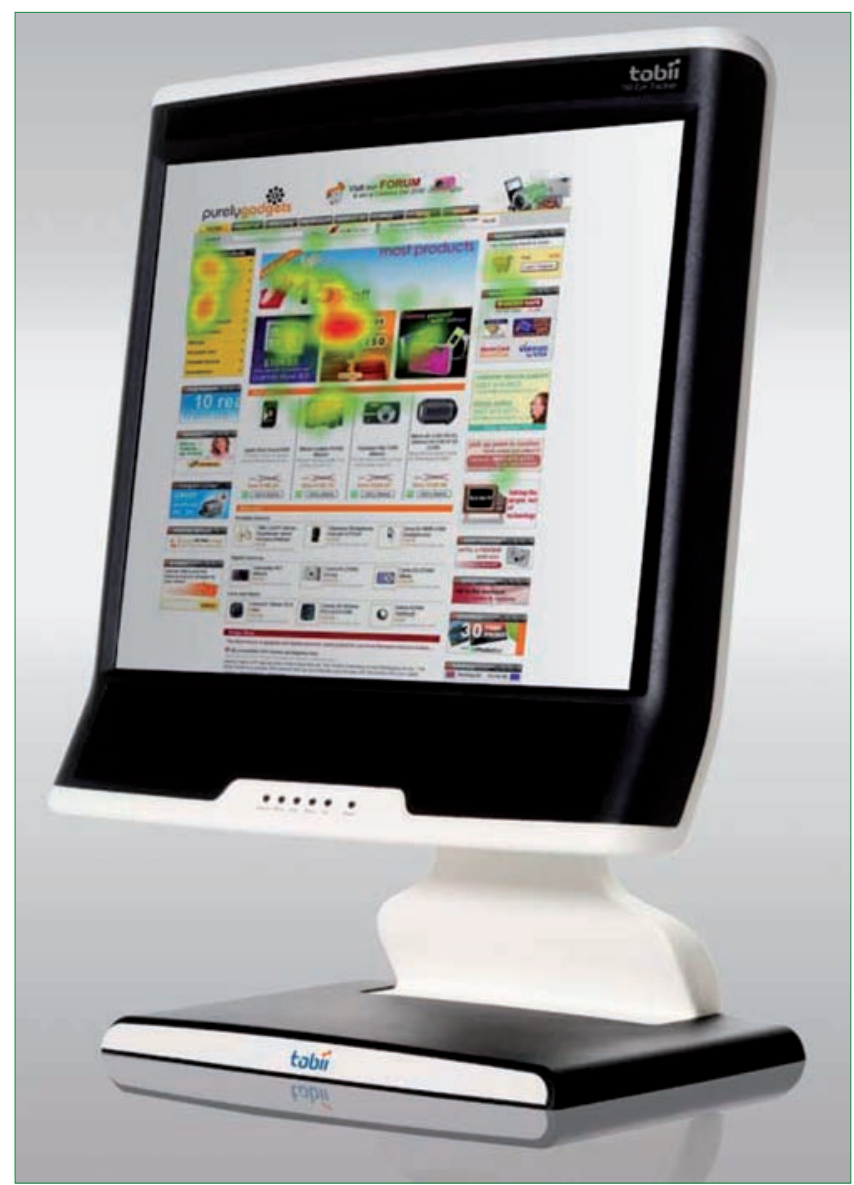

Figura 1. Monitor Tobii T60 para estudios de eye tracking. Ya incorpora la cámara lectora de los ojos.

El ordenador empleado tenía conectado un micrófono y disponía del Tobii T60 como monitor de 17 pulgadas con un sistema ET y una cámara web integrados. Se utilizó como software Tobii Studio versión 1.7.2, que permite extraer y analizar la información captada por el ET.

\section{"Cabe plantearse si la información que se incluye en un pdf debería formar parte de la IA del sitio"}

\subsection{Procedimiento}

Tras la lectura y firma del formulario de consentimiento, los usuarios rellenaron el cuestionario demográfico. Antes de comenzar con la prueba, se solicitó que estudiaran la IA de una web de prueba a modo de entrenamiento. La secuencia de sitios web a analizar se obtuvo de manera aleatoria.

Por cada página web, el usuario tenía que responder a las mismas preguntas de navegación del test de estrés (TEN). Éstas se realizaron siempre en el mis- mo orden, por coherencia con la técnica y para que el procedimiento fuera el mismo en cada caso para todas las webs. A diferencia del TEN, no debían marcar en papel.

La IA se evaluaba también mediante el uso de la técnica de thinking aloud (TA), con lo que los usuarios respondían oralmente a las preguntas de navegación planteadas mientras movían el ratón y la mirada libremente por la interfaz de usuario. Se ha estimado oportuno emplear esta técnica como fuente de obtención de información para determinar los motivos por los cuales no encontraban diversos aspectos de la IA. Se consigue así información cualitativa de los usuarios difícilmente obtenible mediante el empleo de otros métodos, como podrían ser cuestionarios. Por otra parte el empleo de TA junto con eye tracking implica que los usuarios deben mantener la atención en la pantalla.

Según Nielsen (1994), con el método de TA 5 usuarios pueden detectar entre un 77 y un $85 \%$ del total de errores de usabilidad de un sistema. Aunque este trabajo se basa en analizar la IA, se ha de considerar el hecho de tener un número mínimo de participantes para aplicar dicha técnica. También se debe tener en cuenta que el TEN requiere pocos usuarios para detectar la IA subyacente de un sistema web (Instone, 2010).

Para cada pregunta del TEN, los participantes debían analizar la página web mediante la observación hasta localizar el elemento requerido en cada paso. Una vez localizado o cuando el usuario expresaba que le resultaba imposible situarlo, se pasaba a la siguiente pregunta de navegación, repitiéndose el proceso hasta finalizar. Al acabar de evaluar cada sitio web, el participante debía rellenar un cuestionario que analizaba el nivel de usabilidad percibido del sitio en base al cuestionario SUS. Al finalizar éste, se realizó una corta entrevista en la que podía expresar cualquier aspecto que estimara oportuno sobre las webs analizadas.

Un aspecto procedimental destacable es que a los usuarios no les estaba permitido hacer clic mediante el ratón. El método pretende evaluar la respuesta a las preguntas de navegación planteadas sobre las páginas analizadas sin que se desplacen, de modo que no dispongan de otro conocimiento sobre la IA del sitio que no sean aquellos que estén presentes en la página evaluada. En el contexto de este trabajo, el hecho de permitir que los usuarios pudieran navegar por las páginas hubiera desvirtuado su percepción de las preguntas planteadas en el TEN.

En el contexto del método empleado, el uso del SUS, si bien está originalmente planteado como una técnica de evaluación de la usabilidad en la que los participantes interactúan, aquí se propone para la obtención de una medida de la usabilidad del sistema que 
los usuarios perciben tras el análisis de la IA. En este contexto, los resultados del SUS se han de interpretar como estimaciones que proporcionan un valor orientativo sobre la usabilidad de los sistemas analizados.

\section{"El empleo de la técnica thinking aloud (TA) ha permitido detectar las razones por las que los usuarios no encuentran partes de la arquitectura"}

\section{Resultados}

La tabla 1 muestra los resultados de usabilidad percibida del sitio web, o sea, qué sitios son considerados como más usables por los usuarios (mediana), así como el alcance de las diferencias de criterio a la hora de clasificarlos (desviación típica).

Se ha de tener en cuenta que la puntuación que ofrece SUS se basa en la usabilidad percibida por los usuarios sobre todo el sistema, no sobre una única página web concreta. Al ser una puntuación global, se han dado situaciones en las que los comentarios indicaban un grado de usabilidad percibida del sistema relativamente aceptable hasta encontrarse con una página web con una IA diferente que ha modificado la visión que tenían del sistema. También se ha detectado el fenómeno contrario de posible mejora de la valoración del sitio conforme iban aprendiendo a identificar componentes comunes de la IA

\begin{tabular}{|l|c|c|}
\hline \multicolumn{1}{|c|}{ Universidad } & $\begin{array}{c}\text { sUS } \\
\text { mediana }\end{array}$ & $\begin{array}{c}\text { SUS } \\
\text { desviación } \\
\text { típica }\end{array}$ \\
\hline Universidad de Sevilla & 67,50 & 22,76 \\
\hline Universidad de Zaragoza & 87,50 & 26,01 \\
\hline $\begin{array}{l}\text { Universidad de Castilla-La } \\
\text { Mancha }\end{array}$ & 27,50 & 5,40 \\
\hline Universidad de Salamanca & 60,00 & 17,50 \\
\hline Universitat de Barcelona & 72,50 & 25,85 \\
\hline Universitat d'Alacant & 22,50 & 13,28 \\
\hline $\begin{array}{l}\text { Universidad Complutense de } \\
\text { Madrid }\end{array}$ & 87,50 & 5,40 \\
\hline Universidad de Extremadura & 10,00 & 7,36 \\
\hline Universidade de Vigo & 40,00 & 19,40 \\
\hline Universidad de Murcia & 65,00 & 7,50 \\
\hline Universidad de Oviedo & 12,50 & 2,04 \\
\hline $\begin{array}{l}\text { Universidad Pública de } \\
\text { Navarra }\end{array}$ & 43,75 & 37,10 \\
\hline $\begin{array}{l}\text { Universidad de Las Palmas de } \\
\text { Gran Canaria }\end{array}$ & 67,50 & 20,94 \\
\hline Universidad del País Vasco & 32,50 & 7,73 \\
\hline Universidad de Cantabria & 20,00 & 5,14 \\
\hline Universidad de La Rioja & 60,00 & 6,24 \\
\hline Universitat de les Illes Balears & 40,00 & 13,59 \\
\hline Tabla $1 . M e d a n a$ yesvaion & & \\
\hline
\end{tabular}

Tabla 1. Mediana y desviación típica del cálculo de SUS en base a los cuestionarios rellenados por los usuarios del estudio, organizados por universidades

entre diferentes páginas. En este caso, se ha de tener en cuenta que las páginas fueron aleatorizadas, por lo que este posible efecto ha quedado atenuado.

Los resultados correspondientes a las preguntas planteadas en el TEN se sintetizan por sitios web en la

\begin{tabular}{|c|c|c|c|c|}
\hline Universidad & $\begin{array}{c}\% \\
\text { encontrados }\end{array}$ & $\begin{array}{c}\% \\
\text { no encontrados }\end{array}$ & $\begin{array}{c}\% \\
\text { no existentes }\end{array}$ & $\begin{array}{c}\% \\
\text { no aplicables }\end{array}$ \\
\hline Universidad de Sevilla & 85 & 15 & 0 & 0 \\
\hline Universidad de Zaragoza & 94 & 4 & 2 & 0 \\
\hline Universidad de Castilla-La Mancha & 72 & 8 & 19 & 0 \\
\hline Universidad de Salamanca & 72 & 3 & 11 & 14 \\
\hline Universitat de Barcelona & 83 & 2 & 15 & 0 \\
\hline Universitat d'Alacant & 33 & 11 & 56 & 0 \\
\hline Universidad Complutense de Madrid & 71 & 2 & 19 & 8 \\
\hline Universidad de Extremadura & 50 & 11 & 39 & 0 \\
\hline Universidade de Vigo & 88 & 0 & 13 & 0 \\
\hline Universidad de Murcia & 56 & 4 & 40 & 0 \\
\hline Universidad de Oviedo & 42 & 4 & 42 & 0 \\
\hline Universidad Pública de Navarra & 58 & 0 & 42 & 0 \\
\hline Universidad de Las Palmas de Gran Canaria & 71 & 0 & 29 & 0 \\
\hline Universidad del País Vasco & 69 & 6 & 25 & 0 \\
\hline Universidad de Cantabria & 56 & 6 & 39 & 0 \\
\hline Universidad de La Rioja & 67 & 33 & 0 & 0 \\
\hline Universitat de les Illes Balears & 67 & 6 & 27 & 0 \\
\hline
\end{tabular}

Tabla 2. Porcentajes de elementos de la IA según su catalogación 
tabla 2. Ésta se ha construido analizando los vídeos de las sesiones de los usuarios y catalogando sus respuestas en el TEN en base a cuatro criterios: elementos de la IA correctamente identificados, elementos no encontrados, ausentes y no aplicables debido a que en alguna de las webs analizadas correspondía con la de inicio de la biblioteca.

El empleo del eye tracking ha permitido detectar elementos de las páginas analizadas en los cuales los usuarios no fijaban la mirada. Este tipo de información resulta especialmente relevante, puesto que implica que la interfaz de la página no resalta de manera adecuada elementos de navegación. A modo de ejemplo, la figura 2 indica las fijaciones de un usuario sobre la web de préstamo interbibliotecario de la Universidad de Sevilla.

Para resaltar las zonas de la web en las que se ha registrado una mayor fijación de la mirada se emplean los mapas de calor. La figura 3 representa el mapa de calor de los diferentes usuarios que han visitado la web de préstamo interbibliotecario de la Universidad de La Rioja.

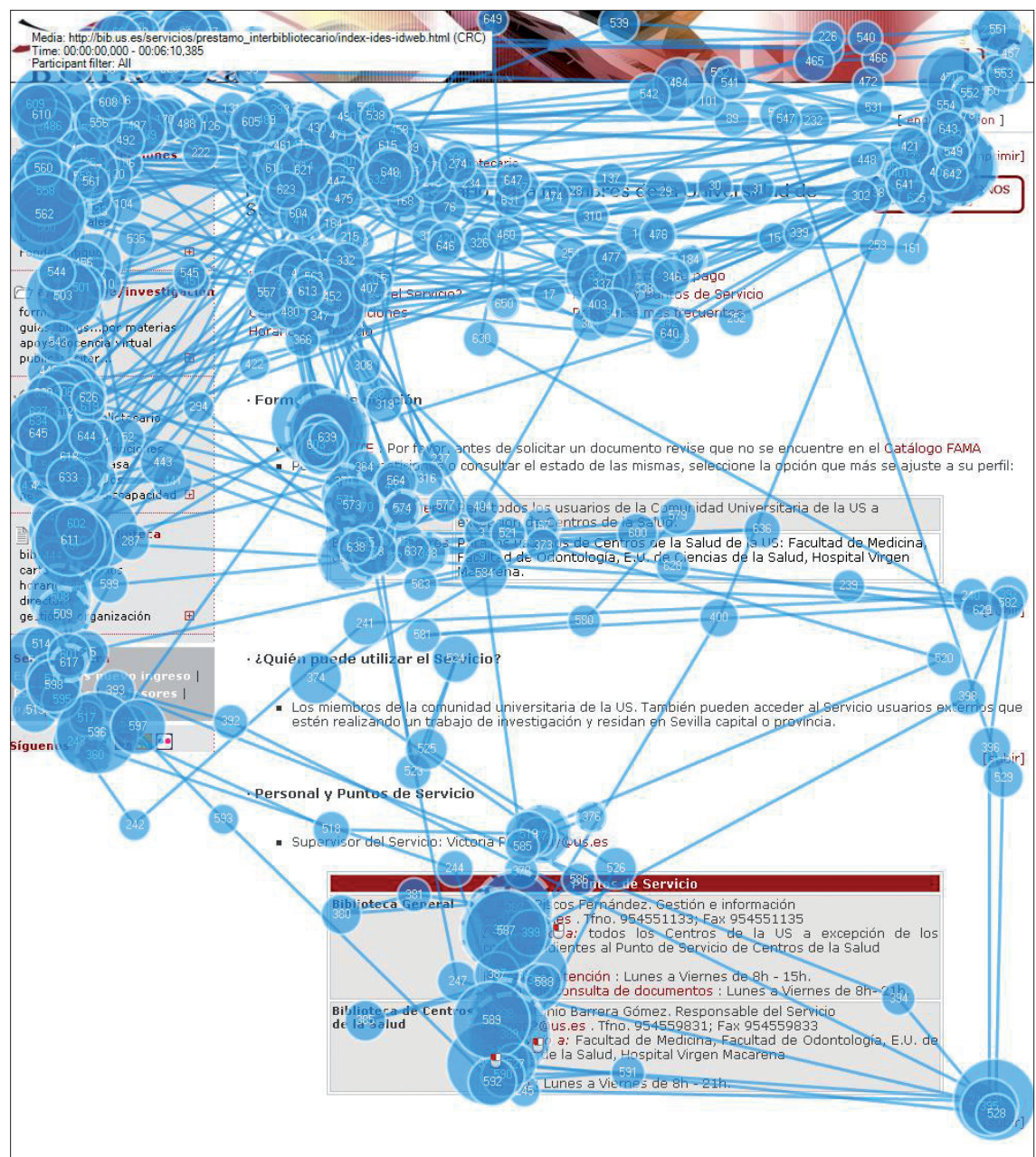

Figura 2. Fijaciones de un usuario sobre la web de préstamo interbibliotecario de la Universidad de Sevilla

Otra función interesante del ET es la posibilidad de definir áreas de interés en las diferentes webs, de tal ma-

nera que a la hora de realizar el análisis de los datos se pueda comprobar el tiempo que los usuarios han estado sobre áreas consideradas importantes. En este caso se han definido áreas de interés en base

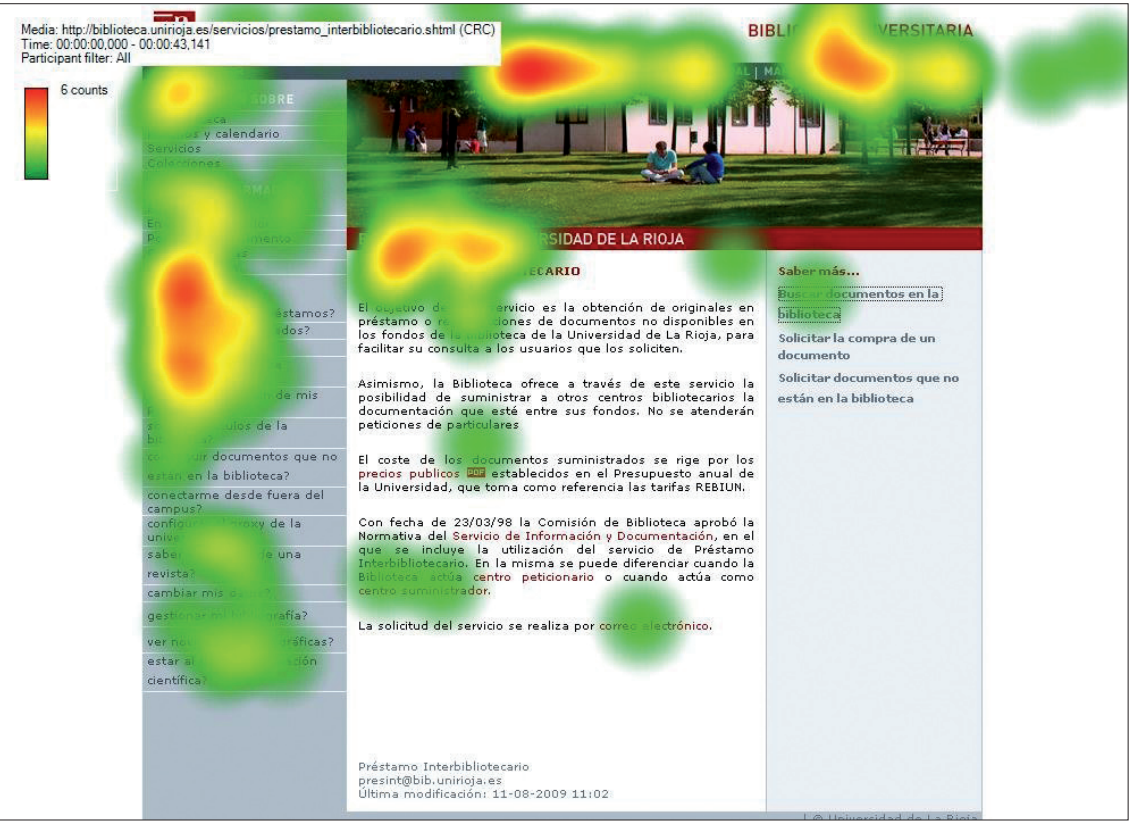

Figura 3. Mapa de calor de los diferentes usuarios que han visitado la web de préstamo interbibliotecario de la Universidad de La Rioja a los elementos que se pedía que los usuarios identificaran mediante el test de estrés de navegación. La figura 4 muestra el análisis de las áreas de interés de la web de acceso remoto a la biblioteca de la Universidad de Las Palmas de Gran Canaria. Resulta apreciable el poco tiempo que los usuarios han empleado para visualizar elementos relevantes de la IA comparado con otros elementos de la página que estaban fuera de las diferentes áreas de interés. Uno de los factores en este caso ha sido el pequeño tamaño relativo de las áreas de interés en comparación con toda la web analizada.

Las preguntas del TEN sobre enlaces, sobre todo en el caso de los externos, han sido en general bien 


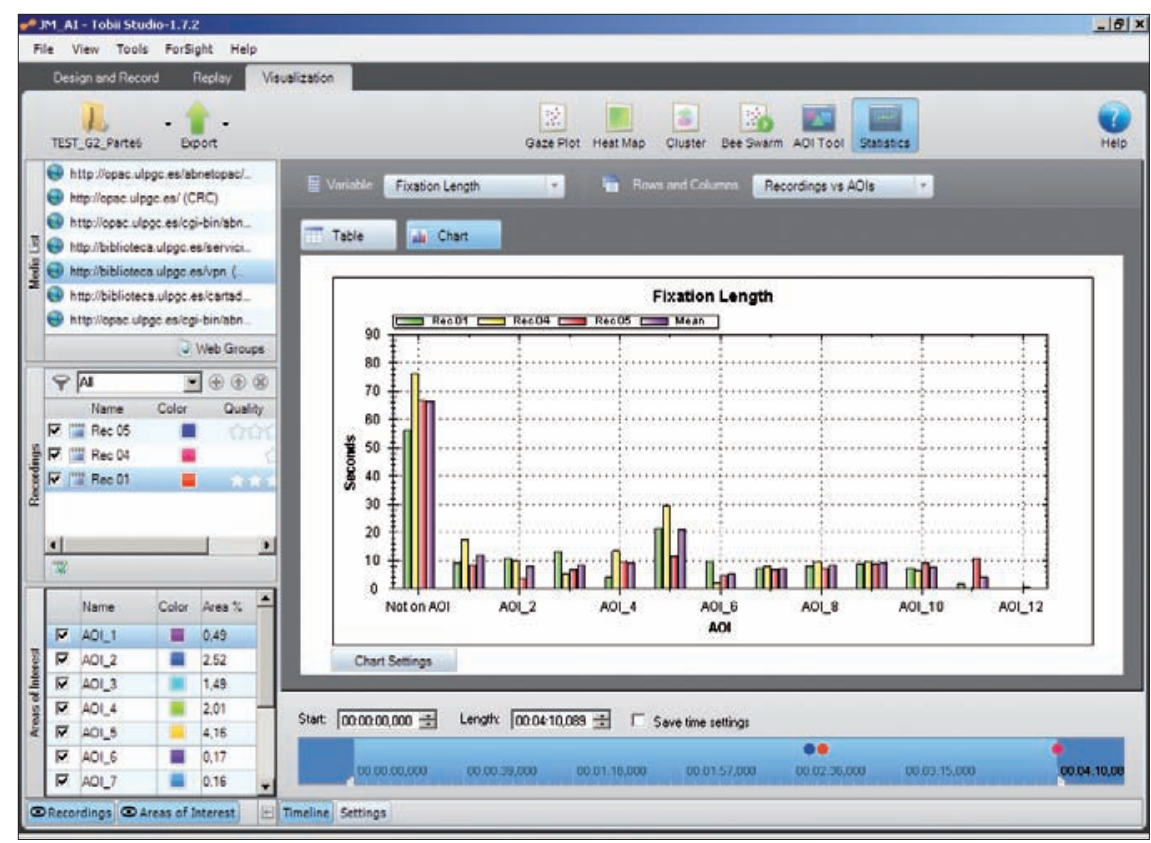

Figura 4. Análisis de las áreas de interés de la web de acceso remoto a la biblioteca de la Universidad de Las Palmas de Gran Canaria

respondidas revisando la ruta de los enlaces. Esta información aparece en la parte baja del navegador cuando se pasa el ratón por encima de aquellos elementos de la página que sean enlaces. Los mayores problemas encontrados se han debido a una incorrecta identificación de la sección actual donde se encuentra la página que se estaba analizando en cada momento. En algunos casos algunos de los enlaces no tenían un objetivo claro o bien podían inducir al usuario a identificarlos correctamente, como el caso del enlace volver en la página del préstamo interbibliotecario de la Universidad de Castilla-La Mancha.

En general se ha observado que dentro de un mismo sitio web, las páginas con un diseño diferente suelen provocar problemas a los usuarios al localizar elementos de la IA. Es porque una página con una IA muy diferente a las ya analizadas, al cambiar toda una serie de elementos comunes a las anteriores, provoca que a los usuarios les resulte más complicado detectar dichos elementos en la nueva IA, dado que no sólo han podido cambiar de lugar, sino que en algunos casos había información de navegación que desaparecía en la nueva IA. Esta situación se ha dado principalmente en los catálogos, donde el cambio de interfaz ha supuesto en muchos casos la pérdida de información relevante de la IA del sitio web de la biblioteca en cuestión. Son cambios que se refieren a la modificación o incluso eliminación de los menús de navegación, sin los cuales desaparece información que permitía que los usuarios respondieran a preguntas concretas del TEN.

En algunas de las webs analizadas (8 casos) la información no se representaba mediante hipertexto, sino que únicamente se podía visualizar mediante documentos en formato pdf. En este sentido, cabe plantearse si la información que hay en el pdf debería formar parte de la IA del sitio en cuestión. Desde el punto de vista del análisis de la IA realizado mediante TEN, dichas páginas representan un serio problema, dado que los usuarios no disponen de información para responder a la mayoría de las preguntas de navegación planteadas en el test de estrés de navegación. La única excepción reseñable es que en unos pocos casos el documento pdf incluía el enlace a la página web de inicio de la biblioteca.

En cuanto a los mecanismos de navegación de los sistemas analizados, gran parte de los usuarios han expresado su sorpresa por la falta de las migas de pan como elemento que esperaban encontrar en algunas bibliotecas.

La interpretación de los resultados debe tener en cuenta que los usuarios tenían gran experiencia de navegación web, por lo que los mecanismos de navegación que han empleado pueden no estar al alcance de perfiles con menos experiencia. En cambio algunos de los aspectos del test requieren de ciertos conocimientos al alcance de perfiles de usuario con experiencia en el uso de bibliotecas virtuales. Como ejemplo cabe destacar que en algunos casos no fueron capaces de reconocer el catálogo de la biblioteca como tal porque el producto empleado, en este caso Papyrus, era desconocido para ellos.

\section{Reflexiones y conclusiones}

El empleo de la metodología propuesta tiene una serie de aspectos a destacar. Por una parte es necesario que sea consistente para poder obtener resultados válidos y comparables entre los diferentes usuarios y sistemas web analizados. Se ha de tener en cuenta que el número de usuarios es un factor clave a la hora de detectar problemas de usabilidad. Se analiza cada entorno web con un total de 3 usuarios, los cuales han detectado la IA de los sistemas analizados de manera casi idéntica entre ellos. Dicho número resulta coherente con los mínimos requeridos tanto por el TEN (Instone, 2010) como por el TA (Nielsen, 1994).

El enfoque metodológico propuesto ha supuesto una serie de aspectos positivos a la hora de realizar el análisis de la IA. El empleo de la técnica del TA ha permitido recoger las valoraciones subjetivas de manera espontánea, lo que ha posibilitado detectar las razones por las que los usuarios no encuentran partes de la 
IA, aspecto que el TEN por sí solo no analiza. También ha sido posible identificar una serie de elementos seleccionados por los usuarios por deducción más que porque las páginas muestren elementos de navegación que delimiten de manera clara los aspectos analizados mediante las preguntas del TEN.

Otro aspecto reseñable es que el disponer de información de navegación dinámica (por ejemplo, que el navegador represente la dirección de los enlaces cuando el usuario pasa por encima de ellos), resulta de especial utilidad para que los usuarios hayan podido responder a las preguntas de navegación. En el TEN, tal y como se encuentra definido, no existe la opción de poder acceder a este tipo de información, ya que se realiza en papel

El ET ha permitido establecer los elementos de las páginas en que los usuarios han fijado su atención y analizar si coinciden con elementos relevantes de la IA. Esta información resulta muy útil a la hora de mejorar el diseño de los sitios web y únicamente se puede obtener mediante el uso de esta tecnología. En este sentido la combinación de dichas fijaciones con la posibilidad de definir áreas de interés dentro del software ha abierto una nueva serie de posibilidades para el análisis.

La aplicación de la metodología planteada implica una serie de costes añadidos respecto al TEN en tiempo de preparación y análisis de los datos recogidos, así como en el equipamiento necesario. El análisis de los datos del ET ha de realizarse de manera cuidadosa. Por ejemplo, las fijaciones han de considerarse en combinación con los resultados del TEN, dado que por sí mismas no son capaces de indicar si el que los usuarios se fijen en partes concretas de la interfaz web redunda en una mejor detección de la IA subyacente.

Aunque la tecnología eye tracking ha evolucionado de manera muy positiva en los últimos años, aún existe un amplio margen de mejora en las herramientas de análisis de los datos recogidos. El software empleado tenía una serie de limitaciones; por ejemplo, para asociar la información recogida mediante ET con webs concretas dentro de la misma herramienta, éstas tenían que mostrarse con el navegador Internet Explorer 6. Este problema resulta de especial importancia si se tiene en cuenta que las webs pueden visualizarse de manera diferente dependiendo del navegador empleado, hecho que puede influir en la detección de elementos de la IA por parte de los usuarios.

Por otra parte, si bien se permite definir áreas de interés de la interfaz de usuario, puede haber elementos de navegación idénticos en diferentes lugares de la interfaz, lo que dificulta el análisis al tener que definir áreas de interés diferentes para elementos con una misma finalidad.
Otro elemento a considerar es el hecho de que en algunos de los sitios analizados el navegador era redirigido a una página diferente para cada sesión de usuario, con lo cual resulta imposible unificar todas en un único análisis conjunto y se han de analizar de manera separada.

El empleo de la metodología planteada es una nueva manera de evaluar la IA de sistemas web en la cual la combinación de diversas técnicas del ámbito de la IPO proporciona diversas fuentes de datos para analizar las causas de los problemas detectados mediante el test de estrés de navegación (TEN).

La primera de las aportaciones que proporciona este trabajo es la realización del TEN en un entorno dinámico como es un navegador web. Ello permite considerar información de tipo dinámico, como la ruta de destino de un hiperenlace, para el análisis de la IA. Este aspecto no es analizable en papel, tal como se define el TEN.

Con el empleo del thinking aloud los usuarios pueden identificar verbalmente problemas relacionados con aspectos concretos del TEN.

El eye tracking permite identificar áreas de interés relevantes respecto a elementos de las páginas web necesarios para responder a las preguntas planteadas en el TEN sobre las que ha sido posible determinar si los usuarios de la prueba han fijado su atención.

Finalmente, aunque sea a un nivel orientativo, el empleo del SUS permite tener una estimación a priori sobre el grado de usabilidad del sistema por parte de los usuarios sin necesidad de realizar una test de usabilidad completo.

\section{Bibliografía citada}

Blackmon, Marilyn H.; Polson, Peter G.; Kitajima, Muneo; Lewis, Clayton. "Cognitive walkthrough for the web". En: ACM Conf on human factors in computing systems, 2002, pp. 463-470.

Brooke, John. "SUS: a 'quick and dirty' usability scale". En: Jordan, Patrick W.; Thomas, Bruce; Weerdmeester, Bernard A.; McClelland, Ian L. Usability evaluation in industry. London: Taylor and Francis, 1996, pp. 189194. ISBN: 0-7484-0460-0.

Instone, Keith. Navigation stress test, 2010.

http://instone.org/navstress

López-Marín, Laura; Méndez, Eva; Sorli-Rojo, Ángela. "Avaluació de 1'accessibilitat i usabilitat dels llocs web de les biblioteques públiques catalanes". Item, 2002, n. 31, pp. 17-51.

http://digital.csic.es/bitstream/10261/8746/1/Avaluaci\%c3\%b3\%20de\%20l $\%$ e2\%80\%99accessibilitat.pdf

Melo-Alves, Fernanda-Maria; Quiroa-Herrera, María-Lourdes. “Análisis y evaluación de sitios web de bibliotecas nacionales: los casos de Brasil y de Portugal". Revista española de documentación científica, 2007, v. 30, n. 2, pp. 199-217.

http://redc.revistas.csic.es/index.php/redc/article/viewFile/379/391

Nielsen, Jacob. "Estimating the number of subjects needed for a thinking aloud test". Intl journal of human-computer studies, 1994, v. 41, n. 3, pp. 385-397. 
Nielsen, Jacob; Pernice, Kara. "Navigation, menus and information architecture". En: Nielsen, Jacob; Pernice, Kara. "Eyetracking web usability". Berkeley: New Riders Press, 2009, pp. 114-141. ISBN: 978-0321498366.

Nielsen, Jacob. Usability engineering. San Francisco: Morgan Kaufmann Publishers, 1993, ISBN: 978-0125184069.

Patalano, Mercedes. "Análisis de los sitios web de las bibliotecas universitarias argentinas". El profesional de la información, 2002, v. 11, n. 2, pp. 102-110.

http://www.elprofesionaldelIAnformacion.com/contenidos/2002/marzo/3. $p d f$

Pérez-Montoro, Mario. Arquitectura de la información en entornos web. Gijón: Trea, 2010. ISBN: 978-84-9704-503-2.

Rosenfeld, Louis; Morville, Peter. Information architecture for the World Wide Web. Sebastopol: O’Reilly Media, 2006. ISBN: 978-0596527341.

Travieso-Rodríguez, Críspulo; Alonso-Arévalo, Julio; Vivancos-Sevilla, José-María. "Usabilidad de los catálogos de las bibliotecas universitarias: propuesta metodológica de evaluación”. Acimed, 2007, v. 16, n. 2. http://bvs.sld.cu/revistas/aci/vol16_2_07/aci04807.html
Vílchez-Román, Carlos. Evaluación de la usabilidad en sitios web de bibiliotecas: una guía práctica, 2004.

http://eprints.rclis.org/10925/

Juan-Miguel López-Gil, Roberto García, Universitat de Lleida. Jaume II, 69. 25001 Lleida.

juanmi@diei.udl.cat

rgarcia@diei.udl.cat

Carolina Navarro-Molina, Rafael Aleixandre-Benavent. Instituto de la Medicina y de la Ciencia López Piñero, CSIC - Universitat de València. Palacio de Cerveró, Plaza Cisneros, 4. 46003 Valencia.

carolina.navarro@uv.es

rafael.aleixandre@uv.es

\section{Cualquier título. Cualquier proveedor Una sola Plataforma.}

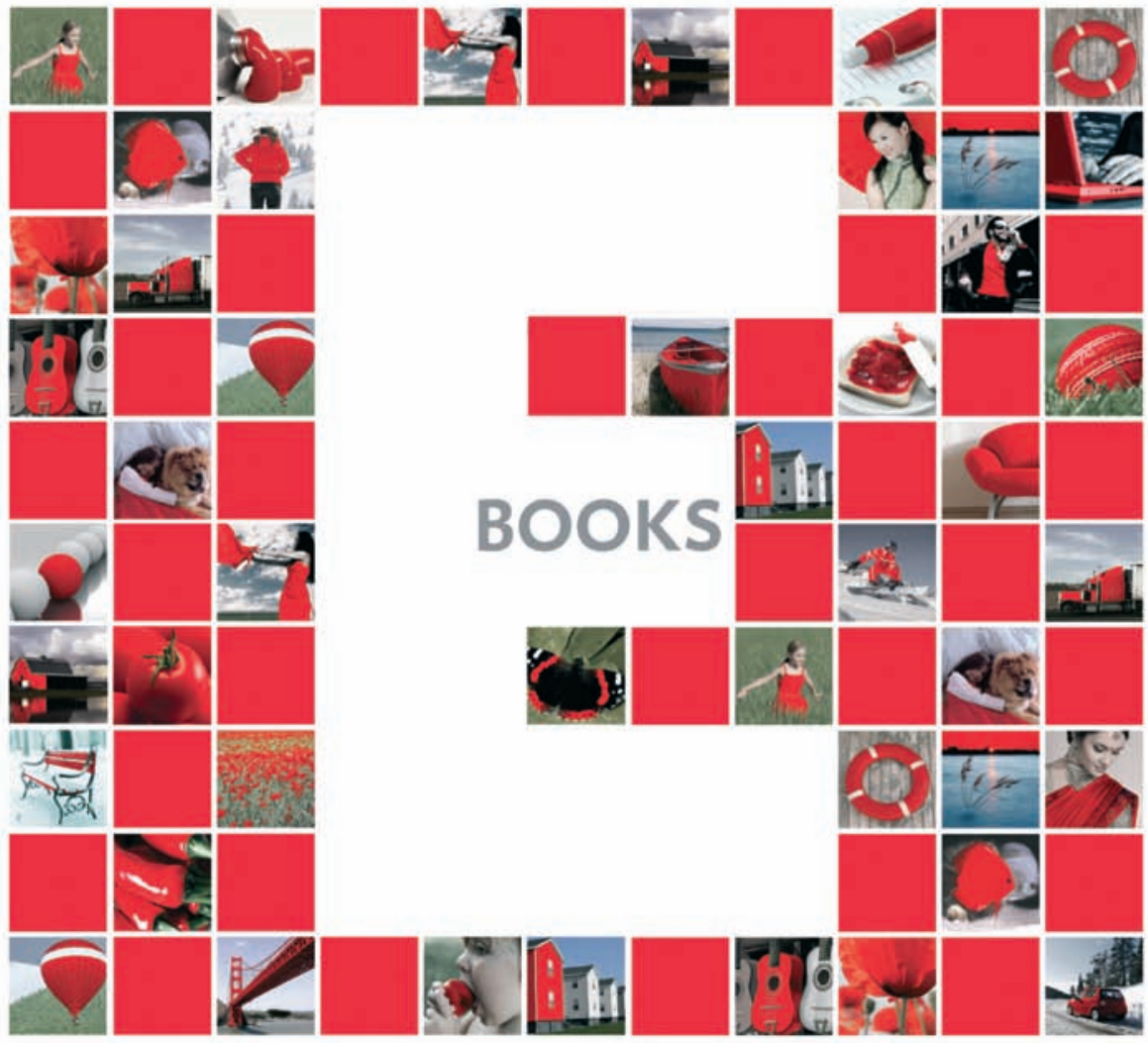

